\title{
PRIMER REPORTE DE LA ESPECIE EXÓTICA PUNTIGRUS TETRAZONA (CYPRINIFORMES: CYPRINIDAE) PARA REPÚBLICA DOMINICANA
}

\section{First report of the exotic species Puntigrus tetrazona (Cypriniformes: Cyprinidae) for the Dominican Republic}

\author{
Alfredo Dalmau-Disla ${ }^{1 \mathrm{a},}$, Patricia Torres-Pineda ${ }^{2}$, Nelvinson de Jesús ${ }^{1 \mathrm{~b}}$ \\ ${ }^{1}$ Escuela de Biología, Facultad de Ciencias de la Universidad Autónoma de Santo Domingo, República Dominicana; ${ }^{\text {la }}$ \\ (D) orcid.org/0000-0003-4771-3473. ${ }^{2}$ Museo Nacional de Historia Natural "Prof. Eugenio de Jesús Marcano", Plaza de la \\ Cultura, Santo Domingo, 10204; (1) orcid.org/0000-0002-7921-3417. "Para correpondencia: dalmaudislaa@gmail.com.
}

\section{RESUMEN}

Se reporta por primera vez para República Dominicana, isla Hispaniola, el pez de agua dulce, Puntigrus tetrazona (Bleeker, 1855) en un tributario del río Haina, provincia San Cristóbal. Se colectaron varios individuos en distintos puntos a lo largo del tributario, sin embargo, no se encontraron individuos de esta especie en el curso principal del río. Se presume que se introdujo intencionalmente y que tiene gran potencial de invasión.

Palabras clave: especie introducida, barbo, ictiofauna, invasor, Hispaniola.

\section{ABSTRACT}

The freshwater fish, Puntigrus tetrazona (Bleeker, 1855) is reported for the first time in Dominican Republic, Hispaniola, in a tributary of the Haina river, San Cristóbal province. Several individuals were collected at different points along the tributary, however, organisms of this species were not found in the main course of the river. It is presumed that it was intentionally introduced and that it is a species with great invasive potential.

Keywords: introduced species, barb, icthyofauna, invader, Hispaniola.

Las especies invasoras se consideran la mayor amenaza para la biodiversidad en ecosistemas aislados geográfica y evolutivamente, como en el caso de las islas (Kairo et al., 2003). La República Dominicana, como nación insular, es particularmente vulnerable a las especies exóticas invasoras, debido a sus frágiles ecosistemas y a la agresividad que presentan estas (Ministerio de Medio Ambiente y Recursos Naturales, 2012), que pueden constituirse en una amenaza a los organismos nativos de la región, bien por depredación, competencia por alimentos y espacio, así como por transmisión de enfermedades (Reaser et al., 2007). Los ecosistemas dulceacuícolas son aún más vulnerables a la presencia de especies invasoras (Dudgeon et al., 2006).

El pez de agua dulce, Puntigrus tetrazona (Bleeker, 1855), se registra por primera vez en un cuerpo de agua natural de República Dominicana, específicamente en "Caño Frío", un pequeño afluente de la cuenca media del Río Haina, en el municipio Villa Altagracia, provincia San Cristóbal ubicado en las coordenadas $\left(18.673303^{\circ} \mathrm{N},-70.181639^{\circ} \mathrm{O}\right)$. Allí se observó a varios individuos nadando en grupos pequeños o bancos. Caño Frío se encuentra alrededor de tierras destinadas a la agricultura y se caracteriza por sus aguas claras, sustrato arenoso, zonas de remanso seguido por pequeños tramos de aguas rápidas, con profundidad máxima de aproximadamente un metro. La mayor parte del arroyo presenta gran abundancia de vegetación palustre y acuática, siendo dominado por la lila de agua (Eichhornia crassipes; Fig. 1). 
El primer avistamiento se realizó el 15 de enero del 2019 por Nelvinson de Jesús, posterior a esto se hicieron tres visitas a la zona, con el fin de detectar la presencia y ausencia de la especie a lo largo del arroyo. Dichas visitas se realizaron el 3 de febrero, 25 de mayo y 25 de agosto del 2019. Para las colectas se utilizaron trasmallos, játicos y trampas nasas para maximizar la captura de la especie.

Se colectó un total 138 ejemplares de $P$. tetrazona, estos se conservaron en alcohol etílico al $70 \%$ y se depositaron en la colección ictiológica del Museo Nacional de Historia Natural "Prof. Eugenio de Jesús Marcano" (MNHNSD-22.10429-10430, MNHNSD-22.10431-10436, MNHNSD.22.1043 7-10464,MNHNSD.22.10465, MNHNSD.22-10466-10478, MNHNSD.22.10479-10548).

El arte de pesca más eficiente para su captura fueron las trampas tipo nasas, cebadas con sardinas. En una nasa se capturó hasta 39 individuos en un periodo de 10 minutos. Se detectaron en 8 de 12 puntos de colecta a lo largo del arroyo, pero no se encontró en el curso principal del Río Haina (Fig. 2).

El "barbo tigre" o "barbo sumatrano" (P. tetrazona), de la familia Cyprinidae, es originario de Sumatra y Borneo. Se caracteriza por la presencia de 3 amplias franjas negras o verdes oscuro en el cuerpo y una cuarta franja que pasa por el ojo, cuerpo amarillo-anaranjado, una aleta dorsal con franjas horizontales negra y naranjas, así como aleta caudal ahorquillada (Fig. 3). Pueden alcanzar una longitud desde los 5 a $10 \mathrm{~cm}$ de largo (Zafra-Trelles et al., 2018). Son generalmente omnívoros, se alimentan de zooplancton, larvas de insectos acuáticos y terrestres, así como invertebrados acuáticos (Shiraishi et al., 1972). Son ovíparos y pueden depositar hasta 500 huevos entre la vegetación sumergida (Scheurmann, 1990; Axelrod \& Sweeney, 1992; Tamaru et al., 1997).

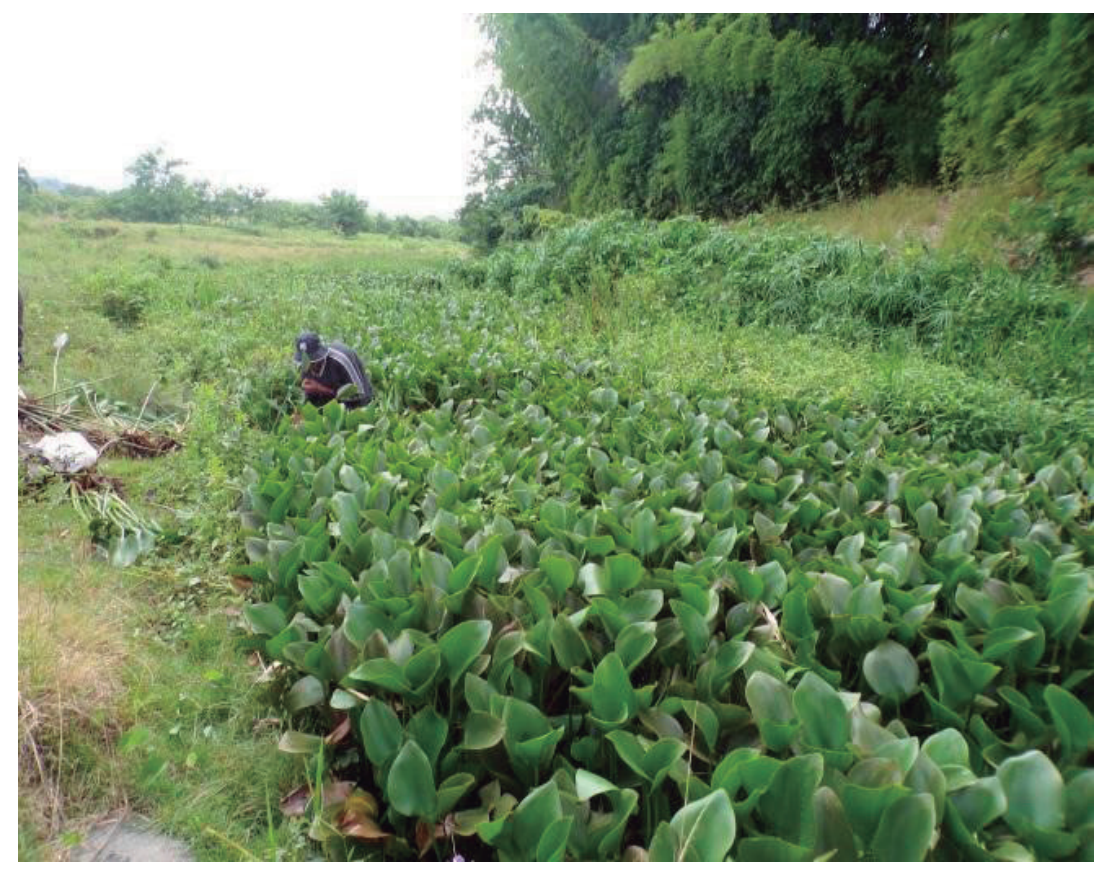

Figura 1. Sección de Caño Frío, dominada por Eichhornia crassipes. Aquí se colectó el mayor número de individuos de Puntigrus tetrazona. Foto: A. Dalmau-Disla. 


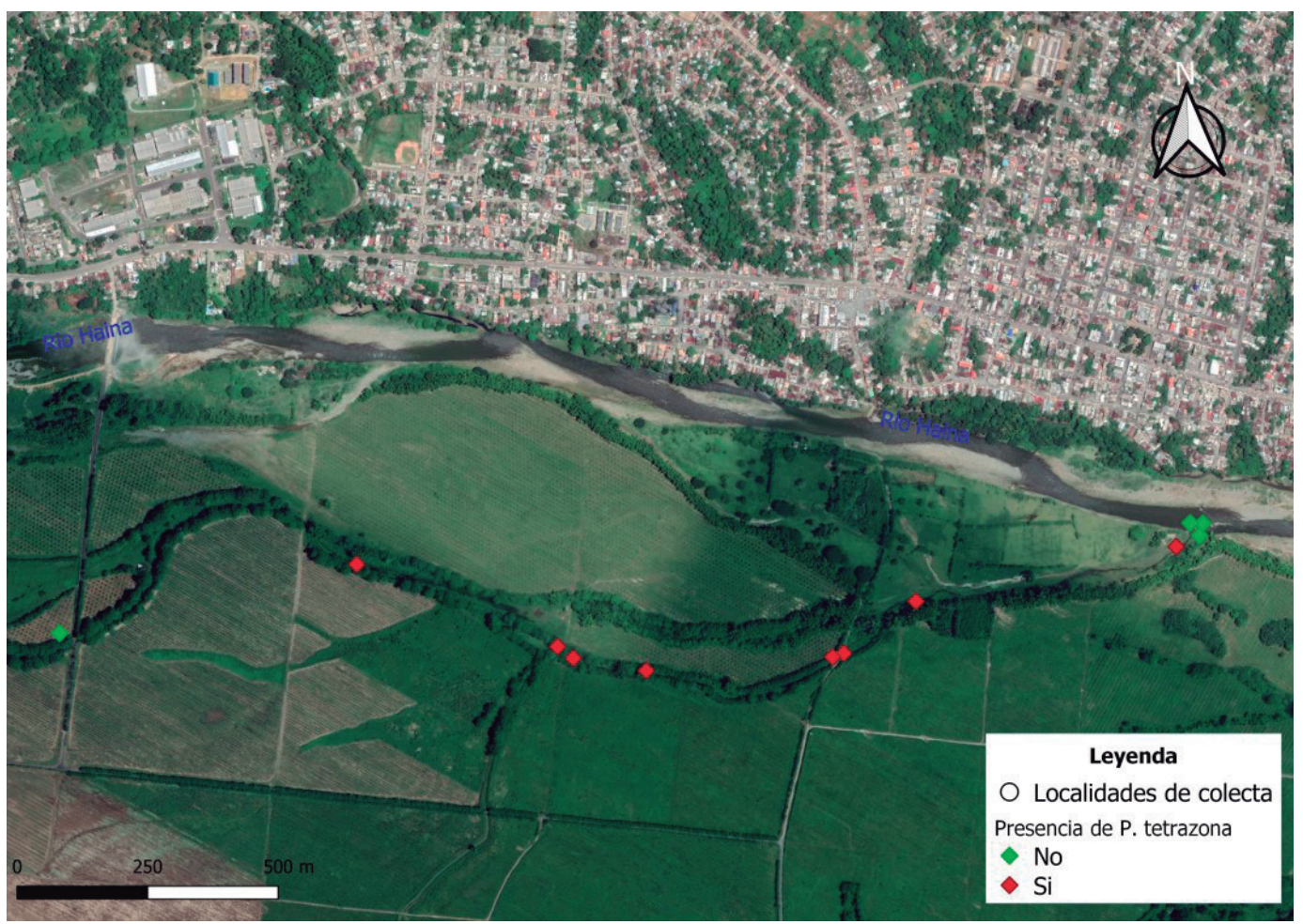

Figura 2. Mapa de localidades de colecta de Puntigrus tetrazona en Caño Frío y Río Haina, Villa Altagracia, República Dominicana. Realizado por Patricia Torres-Pineda.

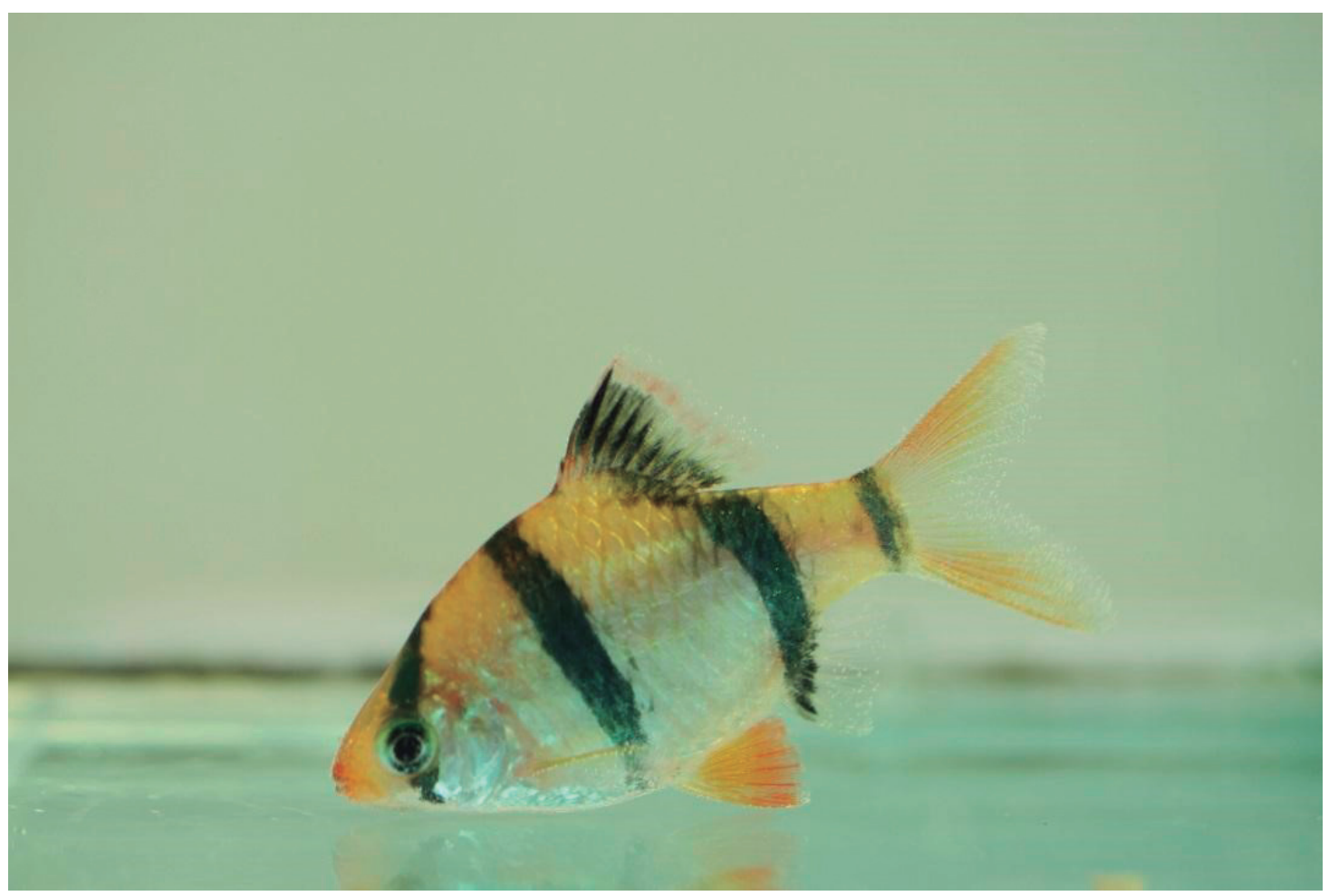

Figura 3. Espécimen de Puntigrus tetrazona (41.31 mm longitud estándar) colectado en Caño Frío, Villa Altagracia, República Dominicana. Foto: Patricia Torres-Pineda. 
Sus colores brillantes, comportamiento gregario y agilidad para formar bancos, así como la facilidad de mantenimiento y reproducción, han hecho de $P$. tetrazona una especie muy popular en el mercado de mascotas (Tamaru et al., 1997), se ha apuntado esta actividad como el principal medio de introducción en muchos países donde ha sido registrado, como en Australia, Colombia, Estados Unidos y Puerto Rico (Nico et al., 2019). Mateo y Balbuena (2011) reportaron que en 2008 y 2009, se importaron entre 100 y 120 individuos de P. tetrazona, para el mercado de mascotas de República Dominicana. Otras especies de tetras y barbos se han importado para el mismo objetivo en los últimos años, incluyendo Puntius denisonii, P. titeya, P. denisi y varias especies del género Paracheirodon (Mateo, J., comunicación personal, 2019).

En varios países donde esta especie ha sido detectada, su introducción se ha relacionado con liberaciones intencionales, principalmente para fines de cría en espacios naturales y posterior captura para su comercialización (Dill y Cordone, 1997; Nico et al., 2019), la locación y características de este arroyo se prestan para tales actividades. El tipo de hábitat conocido para este pez son los arroyos y afluentes con agua relativamente clara, vegetación densa y sustratos de arena y roca de diferentes tamaños (Martínez y Ramírez, 2016), lo que podría explicar el por qué la especie no fue detectada en el curso principal del rio Haina, cuyas aguas son turbias, hay poca o nula vegetación acuática y sustrato rocoso.

La gran cantidad de ejemplares colectados y avistados de estos peces, tanto adultos como juveniles, sugiere que están reproduciéndose en el arroyo. Cabe destacar que, a pesar del esfuerzo de muestreo, no se encontró $P$. tetrazona en los puntos más cercanos que conectan al arroyo con el curso principal del río Haina y otros cuerpos de agua cercanos, lo que podría sugerir que se trata de una introducción reciente y por el momento la especie está concentrada en este arroyo, sin embargo, dadas las características de la especie y su gran potencial de invasión, esta podría esparcirse y colonizar otros pequeños afluentes.

Se ha reportado un comportamiento agresivo de esta especie en acuarios, es conocida por atacar y morder las aletas de otros peces (Martínez y Ramírez, 2016). En el campo se observó a varios individuos de $P$. tetrazona persiguiendo a un individuo adulto de Biajaca (Nandopsis haitiensis, Cichlidae), endémico de La Española, lo que sugiere que la interacción de esta especie con las nativas puede ser negativa, ya que podría perseguir o atacar a otras que habitan en este arroyo, tal como Limia perugiae, Limia versicolor y Poecilia hispaniolana.

Se recomienda un monitoreo a largo plazo de esta especie para conocer su rango de expansión, así como estudiar su biología y ecología en la zona a fin de poder evaluar correctamente sus posibles impactos a la biodiversidad nativa.

\section{AGRADECIMIENTOS}

Agradecemos a Arlen Marmolejo, Omar Paíno y Ángela Guerrero por facilitar parte de los equipos utilizados en el campo. A Jeannette Mateo por sus observaciones y recomendaciones para la mejor presentación de este trabajo. A Merali Morillo, Oniel Álvarez y Francis Reyes por su ayuda en el campo. 


\section{LITERATURA CITADA}

Axelrod, H. R. y M. E. Sweeney. 1992. The Fascination of Breeding Aquarium Fish. T. F. H Publications, USA, 448 pp.

Dill, W. A. y A. J. Cordone. 1997. History and status of introduced fishes in California, 1871-1996. California Department of Fish and Game, Fish Bulletin 178. UC San Diego: Library - Scripps Digital Collection. Disponible en: https:/escholarship.org/ uc/item/5rm0h8qg (accesado: 5/02/2020).

Dudgeon, D., A. H. Arthington, M. O. Gessner, Z. Kawabata, D. J. Knowler, C. Lévêque, R. J. Naiman, A. Prieur-Richard, D. Soto, M. L.J. Stiassny y C. A. Sullivan. 2006. Freshwater biodiversity: Importance, threats, status and conservation challenges. Biological reviews, 81:163-182, doi: 10.1017/S1464793105006950.

Kairo, M. T., A. Bibi, O. Cheesman, K. Haysom y S. Murphy. 2003. Invasive Species Threats to the Caribbean Region: a report to The Nature Conservancy, CABI Bioscience, Egham, Surrey, UK.

Martínez, C. A. y H. M. Ramírez. 2016. Catálogo de peces ornamentales producidos en Morelos con capacidad de ser Especies Exóticas Invasoras (EEI). Elaborado dentro del proyecto GEF, 89333.

Mateo, J. y E. Balbuena. 2011. Caracterización del Comercio de Fauna Acuática Exótica en República Dominicana Durante el Periodo 2006-2010. Proceedings of the 63rd Gulf and Caribbean Fisheries Institute, (63): 93-98.

Ministerio de Medio Ambiente y Recursos Naturales. 2012. Especies Exóticas Invasoras: una amenaza a la biodiversidad. Orientaciones para un manejo adecuado. Santo Domingo, República Dominicana, 36 pp.

Nico, L., P. Fuller, M. Neilson y B. Loftus. 2019. Puntigrus tetrazona (Bleeker, 1855): U.S. Geological Survey Nonindigenous Aquatic Species Database, Gainesville, FL. Disponible en: https://nas.er.usgs.gov/queries/factsheet.aspx?SpeciesID=635 (accesado: 8/29/2019).

Reaser, J, K., L. A. Meyerson, Q. Cronk, M, De Poorter, L. G. Eldrege, E. Green, M. Kairo, P. Latasi, R. N. Mack, J. Mauremootoo, D. J. O’Dowd, W. Orapa, S. Sastroutomo, A. Saunders, C. Shine, S. Thrainsson y L. Vaiutu. 2007. Ecological and socioeconomic impacts of invasive alien species in island ecosystems. Environmental Conservation, 34 (2): 98-111, doi:10.1017/S0376892907003815.

Scheurmann, I. 1990. Aquarium Fish Breeding. Baron's Educational Series Inc. Hauppauge, N.Y.

Shiraishi, Y., N. Mizuno, M. Nagai, M. Yoshimi y K. Nishiyama. 1972. Studies on the diel activity and feeding habit of fishes at Lake Bera, Malaysia. Japanese Journal of Ichthyology, 19 (4): 295-306.

Tamaru, C. S., B. Cole, R. Bailey y C. Brown. 1997. Manual for commercial production of the Tiger Barb, Capoeta tetrazona, a temporary paired tank spawner. Center for Tropical and Subtropical Aquaculture, publication number 129, 50 pp. 
Zafra-Trelles, A. M., M. E. Díaz-Barboza, D. Gil, F. Antonio, V. Alva, K. Alexander, y J. P. E. Colchado Colchado. 2018. Catálogo de peces ornamentales en Trujillo, La Libertad-Perú. Arnaldoa, 25 (2), 757-786, doi: http://doi.org/10.22497/arnaldoa.252.25221Alina.

[Recibido: 03 de octubre, 2019. Aceptado para publicación: 02 de mayo, 2020] 